\title{
ASTROPHYSICAL STATISTICS OF 745 COMPACT GALAXIES NEAR THE GALACTIC NORTH POLE
}

\author{
L. RICHTER, N. B. RICHTER, and P. SCHNELLER \\ Karl Schwarzschild Observatory, Tautenburg, G.D.R.
}

\begin{abstract}
A brief description is given of methods used to find 745 compact galaxies in a 36 sq deg-field around M3. These constitute $24-40 \%$ of all galaxies in this area, brighter than 18.5 magnitude in B. Significant clustering found is related to Zwicky clusters of compact galaxies. Photographic 3-color photometry has been carried out. There seems to be no physical difference between cluster and field objects. The catalogue is to be published elsewhere, together with finding charts and detailed discussion of the photometry and statistics.
\end{abstract}

In a field of 36 sq deg round M3 a search for spherical concentrated (compact) galaxies was made on plates taken with the 134/200/400 cm Tautenburg Schmidt camera.

\section{Method of Selection}

The selection was made independently by two observers (Richter and Richter, 1968), using V or R-plates which were taken at times of good definition (seeing of about $\mathbf{1}^{\prime \prime}$ ). The two observers used different pairs of plates in the stereocomparator. The results were then compared and those objects selected by both observers were listed in the final catalogue. These comprise about $90 \%$ of the whole number. Those objects, which were selected only by one of the observers were once more discussed and inspected on further plates in order either to exclude them or to include them in the final catalogue. So we think the material may be very homogeneous.

\section{Definition of Compact Galaxies: Morphological Criteria}

In order to learn what Zwicky means when selecting compact galaxies we inspected such Zwicky objects on Tautenburg plates. After this process we selected the objects in 3 categories according to the following principles:

(1) Very concentrated: starlike, with a slightly diffuse border only distinguishable by a well-trained observer and very careful comparison with stars of the same brightness in the neighborhood;

(2) Concentrated: nearly starlike, but showing clearly a diffuse border;

(3) Medium concentrated: central part very concentrated like a star. Round this a halo of width not larger than the diameter of the central starlike part.

From the 745 objects selected and inspected for concentration $13 \%$ belonged to category $1,49 \%$ to category 2 and $38 \%$ to category 3 .

In general selection was confined to spherical concentrated objects, to avoid difficulties with photometry by iris photometer. 


\section{Limiting Magnitude of Selection}

The excellent quality of the plates, the large scale $\left(1^{\prime \prime}=0.020 \mathrm{~mm}\right)$ and good resolving power of the plates and light-gathering power of the large telescope allowed us to distinguish compact galaxies with certainty down to the $18.5 \mathrm{mag}$ in $\mathrm{B}$, in some cases even to the 19th mag.

\section{Results}

\section{A. DISTRIBUTION IN THE TEST FIELD}

All 745 objects were mapped according to their positions in the test field. There are obvious apparent accumulations, of which the reality has been investigated by statistical methods used by Meurers $(1957,1968)$ and his co-workers in Vienna for the investigation of star aggregates.

The results are shown in Figure 1. The squares marked with Roman numbers contain aggregates of compact galaxies which are with the highest degree of probability not random.

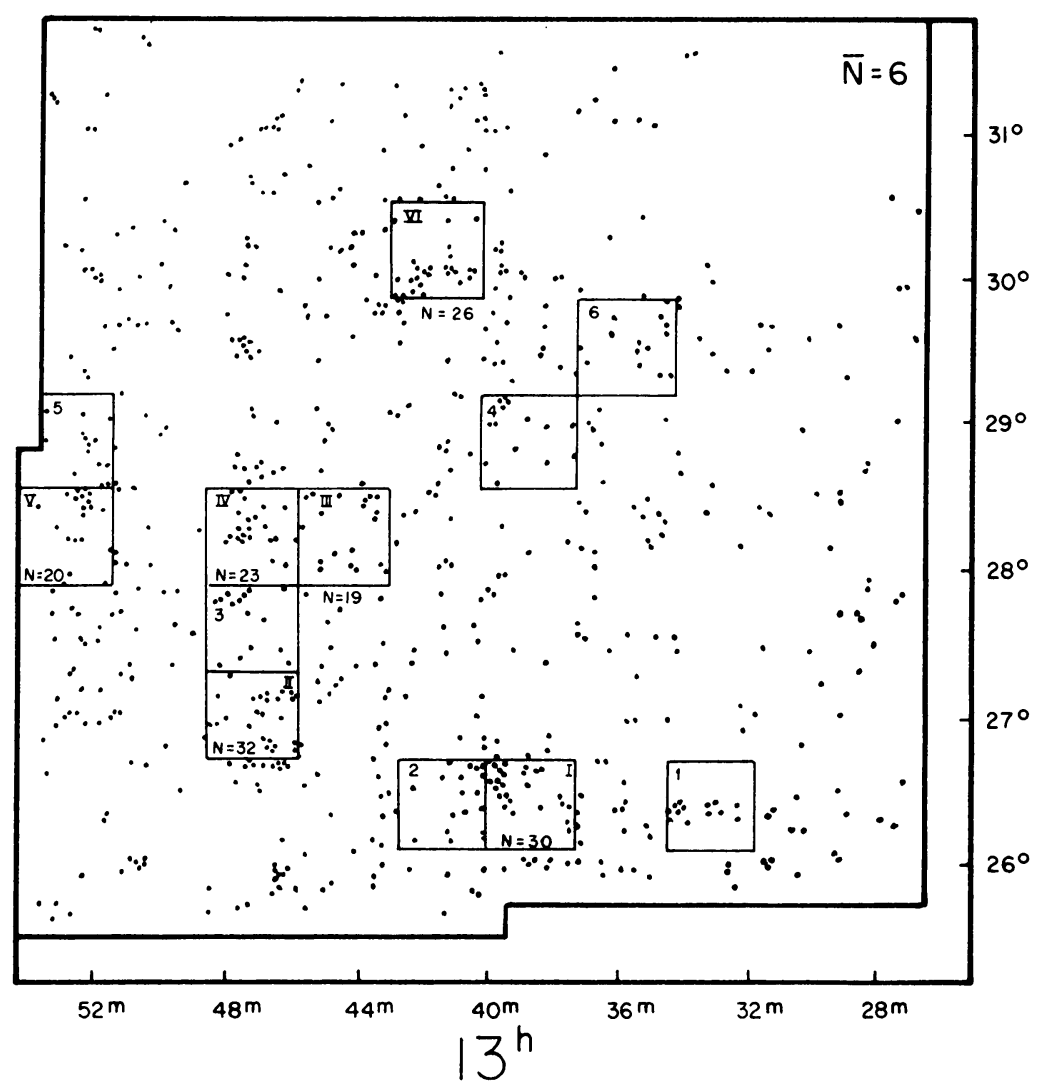

Fig. 1. Positions of 745 compact galaxies in the field round $\mathbf{M}$ 3. The squares enclose aggregates which are with highest probability not random according to statistical tests. 
The squares with arabic numbers contain aggregates which are with great probability also not random.

The next step in the statistical investigation was to compare these aggregates with the positions of clusters of galaxies in Zwicky and Herzog's Catalogue.

In Figure 2 the borders of these clusters are marked on our test field. Clearly all except one our aggregates of compact galaxies are related to normal clusters of galaxies in the Zwicky catalogue.

Seventy-five percent of our objects belong to such clusters, and the remainder are situated in the field between them.

For the clusters $X, Y, Z$ of Figure 2 we determined the proportion of spherical compact galaxies to the total number of galaxies counted in the test field down to the limiting magnitude of selection.

About $40 \%$ of galaxies of all types, visible on Tautenburg plates down to $18.5 \mathrm{mag}$ in $\mathrm{B}$, are compact galaxies according to our selection criteria 1, 2 and 3. If we assume that the objects of category 3 are normal E0 galaxies and should not be counted as compact galaxies there remains still $24 \%$. This seems to be a very high percentage.

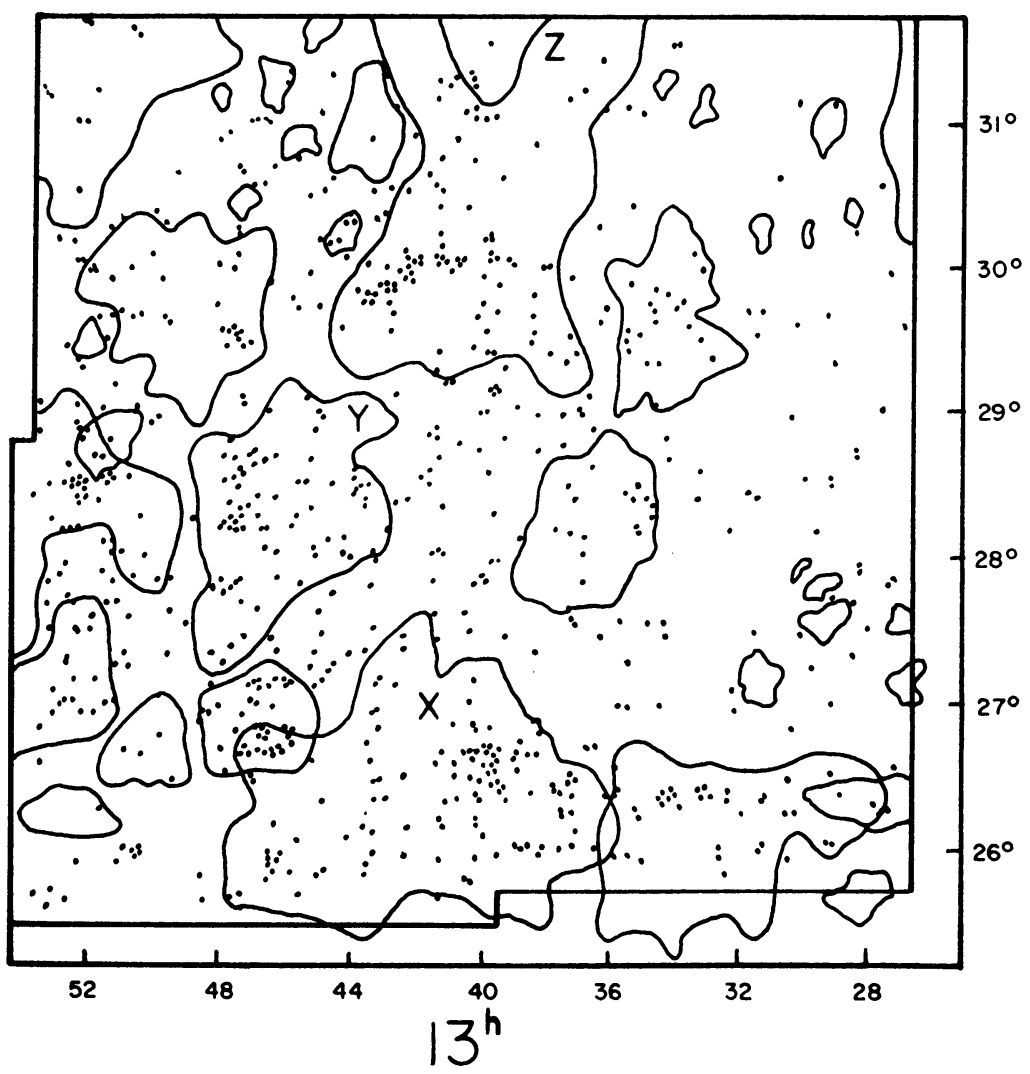

Fig. 2. Positions of 745 compact galaxies and the borders of Zwicky's clusters of galaxies in the test field. 


\section{B. PHOTOMETRIC STATISTICS}

In a former publication (Richter and Richter, 1968) the authors have shown that we must expect systematic errors from integrated photometry of compact galaxies by iris photometry. There is a strong dependence of the brightness of those objects on the limiting magnitude of the plate, especially in system V. The fainter this limiting magnitude the brighter is the measured galaxy. In systems $U$ and $B$ this effect is still small, but in $\mathrm{V}$ the systematic error averages more than $0^{m} .32$ for a difference of 1 mag in the limiting magnitudes of two different plates. There is also a dependence on intrinsic magnitude of the galaxies. The reasons for these effects are known and will be discussed in the final extensive publication.

For the purposes of a photometric catalogue of these objects and for preliminary astrophysical statistics we define quasi-brightnesses (maximal brightnesses) in the different color systems for the plates with the faintest photometric limiting magnitudes.

\section{TABLE I}

\begin{tabular}{lccc}
\hline Brightness (V) & All galaxies & Cluster galaxies & Field galaxies \\
& & & \\
$m<14.00$ & 23 & 16 & 7 \\
$14.00<m<14.99$ & 37 & 25 & 12 \\
$15.00<m<15.99$ & 127 & 87 & 40 \\
$16.00<m<16.99$ & 228 & 163 & 65 \\
$17.00<m<17.99$ & 270 & 191 & 79 \\
$18.00>m$ & 60 & 39 & 21 \\
\hline & 745 & 521 & 224 \\
\hline
\end{tabular}

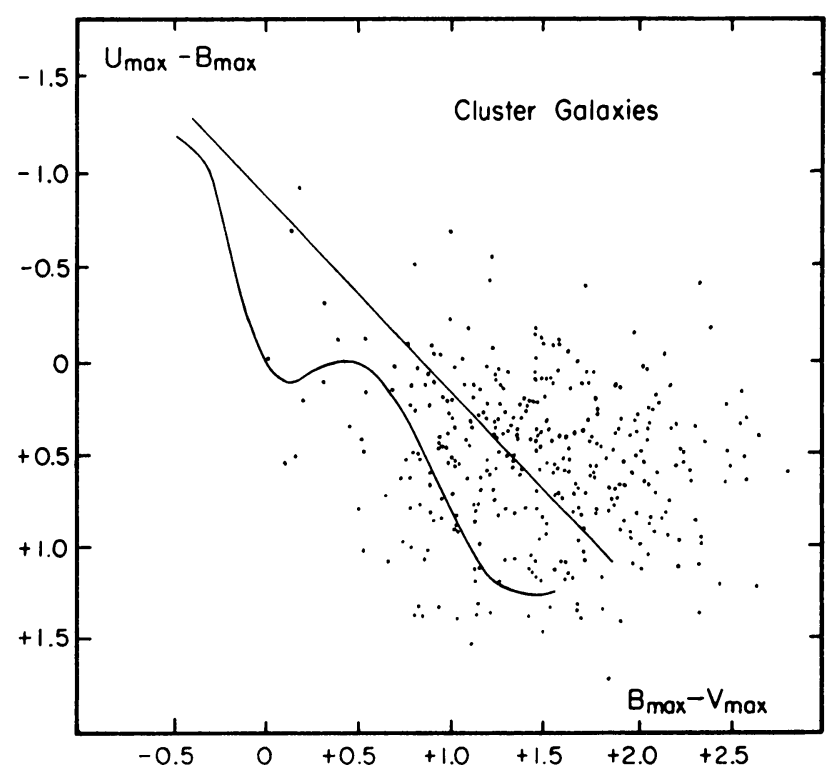

Fig. 3. Two-color diagram of compact cluster galaxies. 
These are the following: U: $20^{m} 0 ; \mathrm{B}: 20^{m} .4 ; \mathrm{V}: 20^{m} .1$.

In Table $I$ are shown the numbers of compact galaxies for different intervals of magnitude for the whole test field.

Special statistics for the large clusters in the field will be published in the final work together with many other statistical investigations. This publication will also include the whole catalogue and finding charts for the 745 compact galaxies.

With some restrictions it is interesting to construct from the quasi-magnitudes of the catalogue two-color diagrams for cluster and field galaxies separately (Figure 3

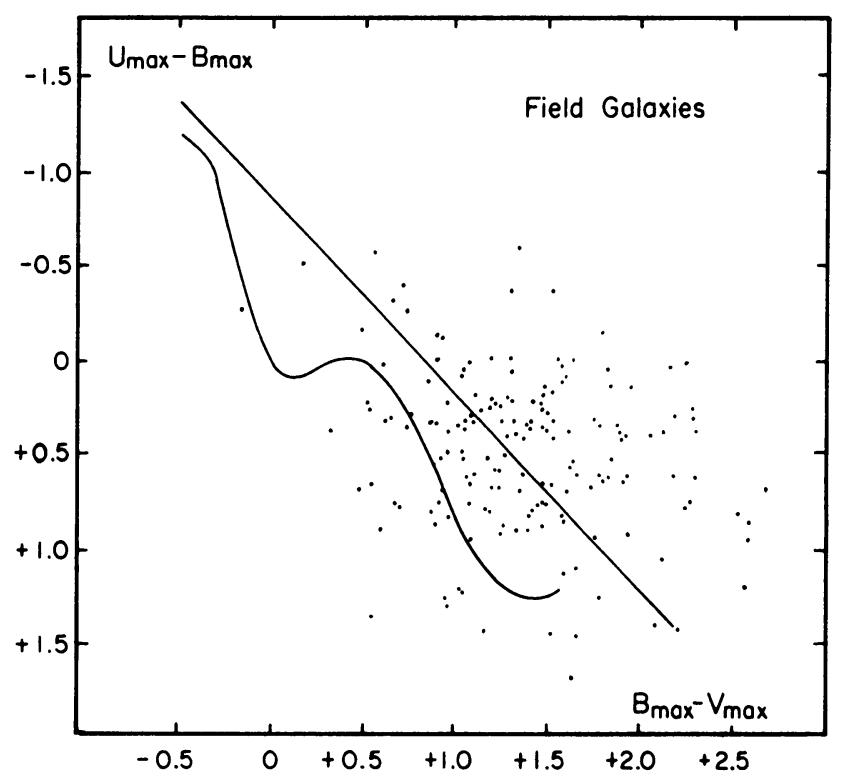

Fig. 4. Two-color diagram of compact field galaxies.

and Figure 4). Of course these diagrams are not representative of the real intrinsic positions of the single objects in the diagrams. The reason we have discussed before. But something can be derived in any case. There seems to be no physical difference between objects in the clusters and in the field around them. And there exists surely a number of blue compact galaxies. All these will be listed in a special investigation to put them at the disposal of spectroscopists.

\section{References}

Meurers, J.: 1957, Veröff. Univ. Sternw. Bonn No. 45.

Meurers, J.: 1968, Ann. Univ. Sternw. Wien No. 5.

Richter, L. and Richter, N.: 1968, Mitt. Karl-Schwarzschild Obs. No. 39. 